Dosier

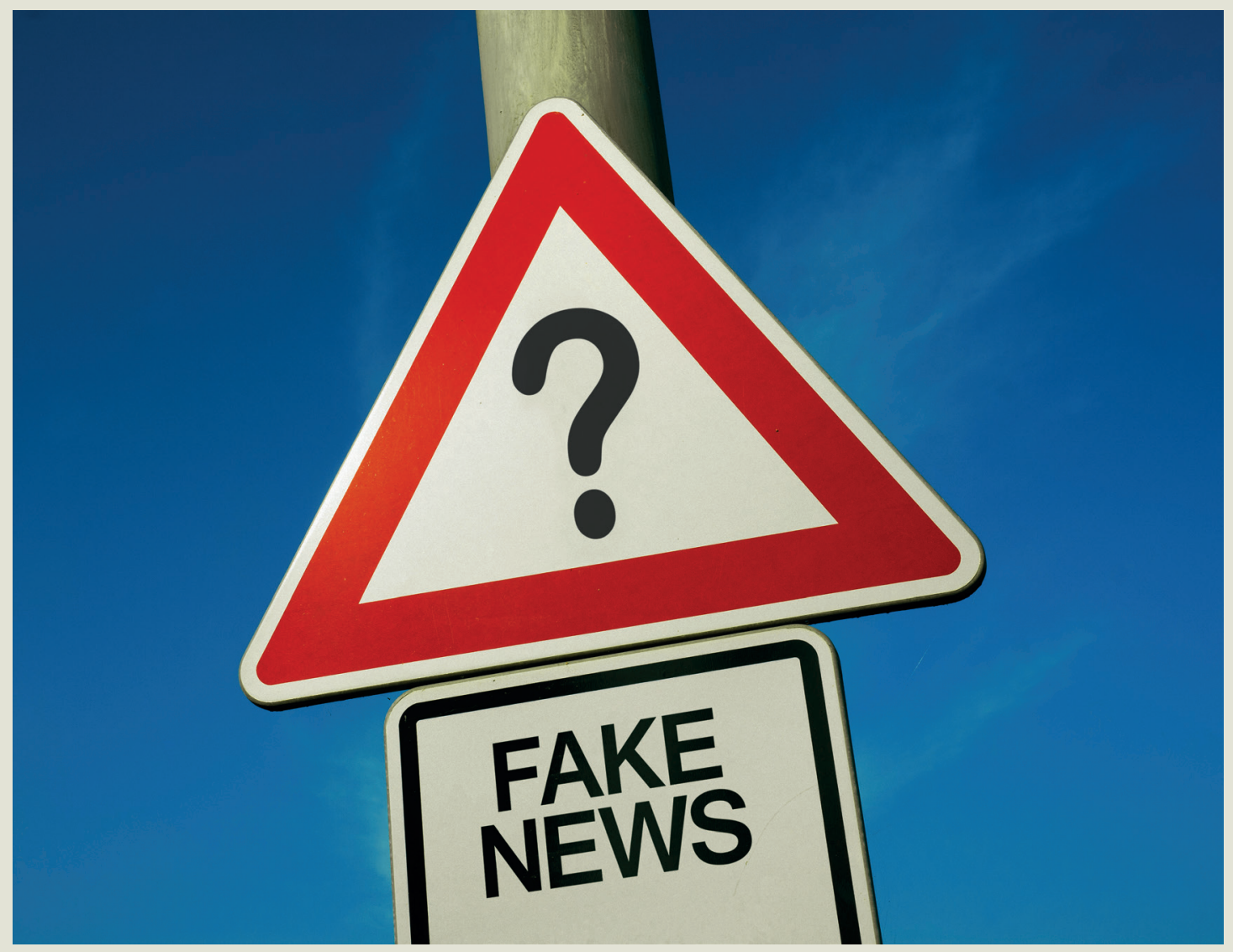





\title{
El periodismo científico y el uso de las emociones en las narrativas noticiosas en la era de la posverdad. Un estudio comparativo entre el Reino Unido y la India
}

Jairo Lugo-Ocando

Universidad de Leeds, Reino Unido

j.lugo-ocando@leeds.ac.uk

\author{
Antje Glück \\ Universidad de Teesside, Reino Unido \\ a.glueck@tees.ac.uk
}

\begin{abstract}
Resumen. En este artículo, examinamos el poder del periodismo científico para atraer y vincularse a las audiencias noticiosas. Observamos cómo las expectativas normativas de ser objetivos e imparciales se han convertido en un obstáculo para la comunicación de la ciencia, a pesar de que son absolutamente necesarias para lograr rigurosidad. El problema es que, aun cuando los periodistas reconocen la necesidad de incorporar elementos subjetivos relacionados con lo emotivo y humano, la naturaleza de la fuente científica impide y limita la capacidad del periodismo de hacer la noticia científica relevante en una era en que las emociones en la comunicación pública son cada vez más relevantes. El artículo refleja los resultados de una investigación desarrollada entre los años 2015 y 2018, y se basa en entrevistas semiestructuradas a 52 periodistas en la India y el Reino Unido, así como en la lectura cerrada de una muestra de noticias científicas producidas en el Reino Unido.
\end{abstract}

Palabras clave: periodismo científico / posverdad / audiencias noticiosas / narrativas / discursos 


\title{
Scientific Journalism and the Use of Emotions in News Narratives from the Post-Truth Era: A Comparative Study Between the United Kingdom and India
}

\begin{abstract}
In the following article, the authors examine the power of scientific journalism to attract and bond with news audiences. They observe how the normative expectations of being objective and impartial have become an obstacle to communicate science, despite being absolutely necessary to achieve rigor. The problem is that, even though journalists recognize the need to incorporate subjective elements related to emotional and human aspects, the nature of the scientific source prevents and limits the ability of journalism to make scientific news relevant in an era where emotions in public communication are increasingly relevant. The article reflects the results of a research developed between 2015 and 2018. It is based on semi-structured interviews with 52 journalists in India and the United Kingdom, and the close reading of a sample of scientific news produced in the United Kingdom.
\end{abstract}

Keywords: scientific journalism / post-truth / news hearings / narratives / speeches 


\section{Introducción}

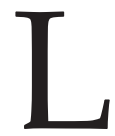

a mayoría de los observadores y académicos hoy en día reconocen que el periodismo actual está en crisis (Russial, Laufer y Wasko, 2015; Peters y Broersma, 2013; Zelizer, 2015). La profesión que tradicionalmente había sido el baluarte de la democracia liberal afronta ahora la "tormenta perfecta" (Jukes, 2013, p. 1). Una combinación de elementos y situaciones que incluye el declive y fragmentación de las audiencias noticiosas, la subsecuente erosión de la economía política que por años sostuvo las finanzas de los medios noticiosos tradicionales, el surgimiento de tecnologías disruptivas y un debilitamiento de la confianza en la profesión periodística como tal por parte del público (Lugo-Ocando, 2015, p. 369). Este vacío, dada la caída en la confianza en el periodismo tradicional, ha disminuido la capacidad de los medios tradicionales para influenciar al público y ha facilitado - en parte- la concreción de la llamada era de la posverdad (Roudakova, 2017; Waisbord, 2018) 1 .

En este trabajo, entendemos la posverdad como un fenómeno en la cultura política donde el público ha perdido la confianza en las fuentes tradicionales de información (léase los periodistas $\mathrm{y}$ medios tradicionales) $\mathrm{y}$, por ende, es susceptible a versiones infundadas de los hechos que presentan afirmaciones sin base, mientras se ignoran las réplicas fácticas. La posverdad difiere de la tradicional disputa y falsificación de la

1 Esta crisis ha creado un vacío de poder importante en la capacidad de los medios noticiosos de movilizar a la opinión pública alrededor de los problemas urgentes de la sociedad y ha socavado su habilidad de mediar en las discusiones políticas, de sentar la agenda pública y de establecer las prioridades por ser discutidas. Particularmente, la consolidación de las redes sociales como primera fuente de información para gran parte del público estadounidense (Moon, 2017) y la pérdida de confianza en los medios tradicionales han tenido un efecto perverso sobre el poder de los medios para dictaminar "de qué hablar" (Cohen, 1963, p. 84) y sentar la agenda noticiosa en general (Conway, Kenski y Wang, 2015; Newman et al., 2017; Neuman et al., 2014). Una era que está caracterizada por la creciente resonancia en el ámbito público de voces populistas que cuestionan los "hechos" y son capaces de establecer "versiones alternativas" a través de "teorías de la conspiración" y otros recursos retóricos (Kalichman, 2009; Oliver y Wood, 2014). Para ser claros, la posverdad, un término que juega con la idea de la relatividad crítica que ofrece la posmodernidad, ha estado siempre presente entre nosotros (Aaronovitch y Langton, 2009; Fenster, 1999). Sin embargo, nunca como ahora había logrado establecerse de forma tan extendida y hegemónica. Quizás, entre sus aspectos más importantes, cabe notar su capacidad de vincularse a las audiencias a través de las emociones y lograr que estas ignoren los hechos y elementos objetivos a favor de explicaciones y versiones "alternativas" (Bakir y McStay, 2017; d'Ancona, 2017; Speed y Mannion, 2017). La elección de Donald Trump como presidente de Estados Unidos, el voto para dejar la Unión Europea por parte de la mayoría británica y el surgimiento de movimientos populistas de derecha e izquierda en varias partes del mundo deben su ascenso en gran parte a narrativas que usan la posverdad como centro de la comunicación política (Allcott y Gentzkow, 2017; Inglehart y Norris, 2016). 
verdad, ya que no ignora la verdad, pero, en cambio, le da una importancia secundaria y cuestiona su validez a la luz de su relación con las élites y el poder (Allcott y Gentzkow, 2017; Waisbord, 2018). En este aspecto, está íntimamente relacionada con las nociones de los sofismas, dado que es una mentira con apariencia de verdad que compite con la verdad fáctica en su habilidad de explicar el mundo y darle sentido al mismo.

Una de las áreas donde el periodismo ha visto más afectada su capacidad de persuasión retórica es la del periodismo científico, donde la posverdad ha creado una serie de problemas, como la desmovilización de la población con respecto al uso de los sistemas de vacunación pública y el socavamiento de la confianza en la autoridad de las ciencias en general, que ha puesto en duda áreas que científicamente son incontrovertibles, por ejemplo, la del calentamiento global y los factores causantes del sida. Consecuentemente, este artículo examina los problemas que el periodismo científico enfrenta en la era de la posverdad, particularmente en lo relacionado con su capacidad de establecer la agenda noticiosa en esa fuente y de movilizar a la ciudadanía hacia la racionalidad científica en las decisiones que toma a diario. En este trabajo, analizamos un área del periodismo que debería ser inmune a la posverdad, dada la naturaleza inalterable de las fuentes y hechos referenciados.

La tesis central es que esta "vulnerabilidad" se puede explicar parcialmente en el contexto histórico del periodismo que, desde su conformación moderna, ha proclamado normativamente ser un paladín de la "verdad", a través de la ideología de la objetividad e imparcialidad periodística (Glück, 2016; Tuchman, 1972; Waisbord, 2013). Como tal, el periodismo aspira a ser científicamente objetivo, en cuanto repercute y moldea la forma en que la noticia es producida y presentada al público ${ }^{2}$.

El hecho de que una gran parte de los periodistas en el mundo anglosajón intenten emular los métodos de las ciencias exactas (Lugo-Ocando y Nguyen, 2017; Ward, 2015) los motiva a desconocer la relevancia de las emociones en la articulación de las narrativas de la noticia (Sillesen, Ip y Uberti, 2015; Soronen, 2017) y, por ende, a desconocer ciertas racionalidades que parecieran ser mucho más relevantes para determinados segmentos de la población que

2 Este rol de "guardianes de la conciencia pública" (Ettema y Glasser, 1998, p. 202), autoasignado por los periodistas mismos, es resultado de la imposición histórica de la racionalidad de la Ilustración como proyecto político. En este sentido, el periodismo moderno es un producto del proyecto político comúnmente denominado de la Ilustración o Época de las Luces (Benchimol, 2015; Christians, 2015). Un proyecto que trata de extender la idea de la búsqueda de la verdad científica al plano político y social (Debarbieux y Rudaz, 2015; Rasmussen, 2014). 
para otros (como lo mostró el caso de la cobertura de la carrera presidencial entre Hillary Clinton y Donald Trump en el 2016).

El periodismo, en este sentido, normativamente hablando, tiende a priorizar el tipo de racionalidades que desconocen la existencia de una "economía de emociones" (Bakir y McStay, 2017, p. 154). De estas racionalidades derivan narrativas noticiosas que asumen que todas las audiencias van a aceptar los hechos y las explicaciones a su valor de cambio. En otras palabras, suponen que los hechos y las narrativas solo pueden tener una explicación única y universal.

Sin embargo, en los últimos años, particularmente después de la invasión militar de Irak en el 2003, cuando la mayoría de los grandes medios noticiosos parecieron aliarse a los gobiernos de George Bush en Estados Unidos y Tony Blair en Gran Bretaña, el público se sintió decepcionado y perdió sistemáticamente confianza en los periodistas, índice que ha disminuido a niveles históricos (Feldman, Huddy y Marcus, 2015; Pew Research Center, 2017; Zuckerman, 2017). Esto se ha traducido en un ambiente donde el escepticismo predomina entre las audiencias noticiosas hacia los medios y los periodistas, en general. Un ambiente dominado por un relativismo posmoderno que abre las puertas a toda clase de narrativas y conspiraciones, que terminan compitiendo por la "credibilidad" en la imaginación pública.

El periodismo científico no se escapa de esta disyuntiva y enfrenta todos los problemas de las demás fuentes (política, economía, crimen, etcétera). Pero, como argumentamos en este artículo, adicionalmente debe lidiar con la naturaleza de una fuente que ha perdido gran parte de su autoridad retórica para convencer al público de lo que es verdad y lo que no lo es. Casos como el escepticismo alrededor de la triple vacuna contra el sarampión, las paperas y la rubeola, en el que millares de padres dejaron de vacunar a sus hijos por los rumores de que esta vacuna podía causar autismo (Begg, Ramsay, White y Bozoky, 1998; Speers y Lewis, 2004), ilustran cómo las teorías de la conspiración pueden llegar a tener un poder mayor de convencimiento que el de la prensa y el de los propios científicos.

Nuestra tesis central sugiere que los periodistas científicos tienden a ignorar y minimizar el rol de las emociones y "racionalidades particulares" en sus narrativas, a pesar de que discursivamente reconocen su importancia. Por consiguiente, en la práctica, sus narrativas dejan de lado las emociones y se concentran en los hechos, siguiendo los cánones de la doctrina de la objetividad periodística. Nosotros sugerimos que en la era de la posverdad esta aproximación se convierte en un obstáculo para ganar la credibilidad y atención del público con respecto a la noticia científica.

Para elucidar el argumento central de esta tesis, entrevistamos a 52 periodistas del Reino Unido y de la India, además de desarrollar una lectura cerrada de varias noticias científicas publicadas en ambos países durante el período de la investigación. Los resultados sugieren que los periodistas se 
debaten entre aspiraciones normativas a la hora de producir la noticia y su propio reconocimiento de la importancia de las emociones. En este sentido, la investigación apunta a un dilema fundamental entre la aspiración de ser objetivo y la necesidad de contar historias con emociones. Igualmente, los resultados muestran que, a pesar de los sustanciales elementos comunes, las dos naciones poseen tradiciones distintas que influyen en la forma en que la noticia científica es presentada.

\section{El periodismo científico en la actualidad}

Comencemos por aclarar que el periodismo científico se enmarca en el área de la comunicación científica. Como tal, es parte de una ecología mediática que incluye los medios de comunicación en general, los sistemas de comunicación profesional relacionados con la ciencia (departamentos de relaciones públicas de universidades, laboratorios y demás, comunicación científica corporativa, etcétera), los museos de ciencia, las enseñanzas de la ciencia en todos los niveles educativos, los sistemas de publicaciones arbitradas, las conferencias científicas y la comunicación política relacionada con la ciencia (campañas electorales, campañas de salud, entre otros). En este contexto, la cobertura noticiosa de la ciencia no puede verse como una práctica aislada, sino que debe comprenderse como una actividad intrínsecamente vinculada a un andamiaje complejo que permite tanto el intercambio de ideas entre las comunidades de científicos, como entre estas comunidades y el mundo exterior.

Es en esta última parte del andamiaje en donde el periodista actúa como mediador conectando a los científicos con el mundo exterior; allí podemos ubicar al periodismo científico como una actividad interpretativa y traductora. Por un lado, como lo ha sugerido Zelizer (1993), más que definir el periodismo como una profesión homogénea, habría que entender a los periodistas como parte de una "comunidad interpretativa" que comparte discursos y visiones del mundo, generando interpretaciones colectivas de la realidad social (p. 219). Según este análisis, los periodistas crean constantemente la realidad social por vía de las narrativas y regímenes discursivos que incorporan en sus reportajes (Elías, 2008; Sánchez Lobato y Hernando, 2018).

Por otro lado, los periodistas también son traductores de lo observado. Esta traducción se lleva a cabo en los términos de referencia que ellos perciben como comunes y accesibles para sus audiencias. Mientras muchos idealistas tienden a creer en una forma de democracia conversacional y deliberativa, el periodismo tradicional abraza, en cambio, una comprensión agregada de la democracia (Anderson, 2011, p. 542), según la cual hay que informar al público desde arriba. Esta visión, donde la diseminación de la información se produce de manera jerárquica, todavía predomina hoy en día, en la era de las redes sociales. Al hacer esto, los periodistas automáticamente 
se ven a sí mismos como guardianes y reguladores de las ideas que se comunican, convirtiéndose así en mediadores entre la producción científica y el imaginario público (Bauer y Bucchi, 2008; Dunwoody, 2014).

El asunto se problematiza aún más porque, al contrario de las posturas normativas que sugieren que el periodista es un mediador objetivo y neutral, los periodistas tienden más bien a enmarcar el contenido noticioso que ellos generan en el contexto de sus propias creencias, valores e ideologías profesionales (Deuze, 2005; Fowler, 2003). Tales creencias, valores e idolologías constituyen la base de las interpretaciones colectivas y, por consiguiente, definen los paradigmas de conocimiento alrededor de los cuales los periodistas determinan lo que ellos consideran como la "verdad objetiva" (Hermida, 2012, p. 659).

Esta aproximación a la realidad social no solo es característica de la práctica periodística en Estados Unidos y Europa Occidental, sino que, además, representa una aspiración normativa realmente global, como lo han señalado las encuestas mundiales entre periodistas elaboradas por el proyecto de The Worlds of Journalism Study (WJS, 2018). Este es el caso incluso del periodismo científico, que reclama normativamente para sí un rol de mayor objetividad y neutralidad, dada la naturaleza de la fuente científica a la que se presume por definición neutral y apolítica. Sin embargo, al igual que la cobertura de otras fuentes noticiosas, la comunicación de la ciencia está sujeta también a las prerrogativas políticas, económicas y sociales que determinan la forma en que la noticia, en general, es enmarcada.

En este sentido, el periodismo científico se practica en el marco de un régimen discursivo específico. Este es definido por Gee (1999) como el marco que establece los términos de referencia para las discusiones y narrativas que se dan en un área determinada en la sociedad, a los que él se refiere como "conversaciones" (p. 49). Uno de los elementos más importantes de estos regímenes es que establecen los parámetros para lo que es aceptado o no dentro de las conversaciones públicas. Por ejemplo, entre esos parámetros, se encuentra la idea de que la ciencia es racional-lógica $\mathrm{y}$, por consiguiente, es una conversación pública, mientras que la religión es una conversación a la que se le considera irracional-emocional y que, por lo tanto, debe mantenerse en privado.

En consecuencia - para continuar con el ejemplo-, para el periodismo tradicional, los argumentos de la religión solo tienen validez en la esfera privada. La narrativa, entonces, es una en la que todo lo "religioso" no puede sino ser calificado como metafísico y, por ende, carece de legitimidad racional en la discusión científica, incluso en lo referente al aspecto moral. De esta forma, para citar un caso, la discusión pública en los medios sobre la guerra en Irak se sustentó fundamentalmente en los "argumentos científicos". En este sentido, los periodistas examinaron el argumento en favor de la guerra centrándose en la posibilidad de que Sadam Husein poseyera o no armas químicas 
de destrucción masiva. Esto sucedió a pesar de que las mayores protestas contra la invasión de Irak en el 2003 fueron organizadas precisamente por las distintas Iglesias y grupos religiosos sobre una base moral. Es decir, la prensa ignoró o reportó de forma inadecuada las respuestas morales de la sociedad civil, según las cuales lanzar un ataque contra Irak causaría la muerte de muchísimas personas y Occidente no tenía bases morales para intervenir.

De cara a aquello, la gran prensa reportó burlonamente esta oposición, describiéndola en muchos casos como un movimiento de abraza árboles (tree huggers, en inglés), mientras que los argumentos en contra de George Bush, Tony Blair y el dosier que justificó la guerra se tildaron de "irracionales" (Luther y Miller, 2005; Murray, Parry, Robinson y Goddard, 2008), ya que carecían de elementos "científicos". La presentación de un dosier científico -que terminó siendo altamente cuestionado- impuso una narrativa científica que logró desplazar de la discusión pública los referentes éticos y morales que rechazaban la guerra.

En este sentido, la ciencia se superpuso a la religión como un régimen discursivo que aspira a ser objetivo y científico, y para el cual la única realidad verdadera es aquella que es tangible y cuantificable, donde ni emociones ni creencias morales tienen lugar en el análisis (Glück, 2016; Martinisi y LugoOcando, 2015). La forma en que los grandes medios occidentales dieron cobertura tanto al conflicto como a la sociedad civil que se oponía a la guerra en el 2003 tuvo - como hemos visto en los años subsiguientes - un efecto perverso en los niveles de credibilidad en los políticos, los medios noticiosos y la argumentación científica, en general. Allí se encuentran las semillas de la falta de credibilidad que hoy aqueja al periodismo y que ha permitido el surgimiento y expansión de la posverdad.

\section{Retos científicos}

Vale aclarar que el periodismo científico no ha tenido que enfrentar en tiempos recientes el fenómeno Irak, en cuanto no ha habido un momento específico que haya marcado particularmente el declive de su credibilidad entre el público. Por el contrario, esta se ha ido erosionando paulatinamente gracias a la complejidad de la relación entre las actitudes públicas y la producción científica en el contexto de la sociedad (Sturgis y Allum, 2004, p. 55). Hasta cierto punto, uno puede argumentar que la erosión en la credibilidad de la fuente científica precede a la era de la posverdad. Esto se debe a que para la fuente científica el problema de la credibilidad es complejo y está íntimamente relacionado con el contexto político, algo que no siempre se reconoce en esa área. Por ejemplo, una investigación del Pew Research Center en Estados Unidos revela que, aunque la credibilidad de los científicos permanece más o menos estable en las últimas décadas, es mucho más baja entre los sectores conservadores de derecha que entre los sectores progresistas de 
izquierda (Rainie, 2017). Este resultado está vinculado a los niveles de educación y al grado de penetración de la instrucción pública.

Pero los científicos son solo una parte de la ecuación y una de las muchas voces en la ecología mediática que constituye el sistema de la comunicación científica, el cual, como argumentan Conway y Oreskes (2010), está influenciado por una cantidad deactores corporativos que comprometen los niveles de transparencia y rigurosidad. Este sistema de comunicación corporativa profesional significa que en poquísimas ocasiones los científicos se comunican directamente con el público o con los periodistas. Hoy por hoy, los centros de producción científica -laboratorios, universidades, centros de investigación, etcétera- poseen equipos enteros de relacionistas públicos que generan y gestionan las comunicaciones de estas entidades (Bauer y Bucchi, 2008; Linstead, 2015).

Este proceso en que las instituciones han politizado sus dinámicas y las han adaptado a la lógica de la cobertura mediática se conoce como mediatización, y no debe confundirse con el concepto relacionado con la percepción por vía de los sentidos. El término mediatización fue adoptado hace ya varios años en el área académica por los investigadores que trabajaban sobre las tecnologías de comunicación. Según este análisis, las instituciones políticas en los últimos años han adaptado sus dinámicas a las de los medios. Con ello se sugiere que las instituciones de la sociedad, incluyendo aquellas que producen ciencia, han adaptado su vinculación con la sociedad a las dinámicas de los medios de comunicación. De allí que ahora veamos que universidades, centros de investigación, corporaciones farmacéuticas - por nombrar algunas - tengan ejércitos de relacionistas públicos y especialistas de la comunicación para lidiar con una relación sociedad-institución que hoy en día está altamente mediatizada.

En consecuencia, los medios pasan a difundir los productos simbólicos de la esfera política que han ampliado la propiedad pública de los actores políticos y de las acciones o acontecimientos que ocurren en la esfera pública. Los medios de comunicación ahora son los principales productores de cultura mediática, y posibilitan, fuera del ámbito de la experiencia directa, la apropiación de los hechos y la construcción de significados que permiten la comprensión y explicación del mundo exterior. Por consiguiente, es posible argumentar que gran parte de los acontecimientos de la esfera pública están mediatizados por la lógica y las funciones de los medios de comunicación (Hjarvard, 2008; Lundby, 2009). La ciencia, como hemos explicado arriba, enfrenta también esta situación y hoy es imposible pensar en la generación del conocimiento científico sin un componente mediático importante.

Tomando en cuenta estas dos grandes vertientes, la del régimen discursivo predominante y la de la mediatización, se puede argumentar que la forma en que el periodismo científico reporta está altamente condicionada por factores externos que determinan el tono y 
contenido de lo que se dice y cómo se dice. Más allá de la aspiración normativa que sugiere que los periodistas científicos informan objetivamente sobre la producción científica, el contexto más bien apunta hacia una cobertura altamente definida por esas vertientes. En este sentido, puede aducirse que hay un aparato científico-industrial que define la práctica del periodismo científico.

Como consecuencia de esto, la crítica hacia el periodismo por parte de la comunidad científica se centra mayoritariamente en la falta de rigurosidad (Reed, 2001, p. 279). Según esta postura, el problema del periodismo científico es que no reporta con suficiente rigor los acontecimientos y eventos científicos (Brechman, Lee y Cappella, 2009, p. 453). De allí que muchos propusieran el desarrollo integral de la comunicación científica donde el investigador adquiriera las herramientas comunicacionales para vincularse directamente con la comunidad y difundir el conocimiento generado. Este ha sido el eje central de la política de comunicación científica de la mayoría de los gobiernos y centros de investigación alrededor del mundo.

A pesar de ello, esta aproximación no ha dado los resultados esperados. Primero, porque la especialización en el periodismo científico no garantiza la calidad. Como lo han demostrado varias investigaciones previas, un reportero con preparación en biología, por citar un ejemplo, sí puede cubrir la fuente de biología y medicina quizás con mucha mayor rigurosidad que un periodista generalista. Sin embargo, una vez que el periodista-biólogo trate de informar sobre otra área como física o química, estará en la misma desventaja que el generalista. Igualmente, este modelo desconoce la economía política de los medios noticiosos que difícilmente pueden sustentar este grado de especialización entre su personal.

Segundo, porque si bien la transición de científicos a comunicadores no es de por sí problemática, la de científicos a periodistas sí lo es. La comunicación científica, como hemos dicho arriba, abarca una cantidad de áreas como la industria del entretenimiento, los museos, los programas educativos de extensión, entre otras. Pero estas vías de comunicación requieren aproximaciones y metodologías distintas en sus estrategias de vinculación con el público. En este sentido, el rol del periodismo científico no es solo el de difundir la ciencia; esto constituye apenas una parte de la labor. Contrario a limitarse a la divulgación científica, el periodista debe escudriñar a la ciencia del mismo modo que se hace con otras fuentes noticiosas (Hibberd y Nguyen, 2013; Nguyen y Mcllwaine, 2011).

Entonces, el ejemplo por seguir para la mayoría de los científicos -y del cual toman referencia constante a la hora de imaginar el tipo de periodismo científico que "ellos" desean- es el de Carl Sagan (1934-1996), indudablemente uno de los divulgadores científicos más importantes y efectivos en el uso de los medios del siglo xx y quien, además, promovió importantes campañas políticas apoyándose en la ciencia. Entre sus logros destaca su lobby efectivo contra la 
proliferación nuclear durante la era de Ronald Reagan (1911-2004), que advertía al mundo de un posible "invierno nuclear", así como su exitosa serie de televisión, que se convirtió en una inspiración para millones de niños y jóvenes que se volcaron a la ciencia luego de verla (Badash, 2009; Shermer, 1999).

No obstante, menos conocido $y$, sin embargo, mucho más pertinente es el caso del periodista del Chicago Tribune, John M. Crewdson, quien puso en evidencia las controversias alrededor de quien había logrado aislar el síndrome de inmunodeficiencia adquirida (VIH/ sida) en una serie de reportajes que le harían merecedor del Premio George Polk de reportajes de medicina. Su trabajo obligó al Comité del Premio Nobel de Medicina a retirarle el galardón al Instituto Nacional del Cáncer de Estados Unidos y otorgárselo al Instituto Pasteur de París, que, además, recibió millones en indemnización. El trabajo de Crewdson es, sin duda, el paradigma que resulta más relevante y convergente con las funciones del periodismo y su rol en la sociedad.

Tercero, la falla fundamental de la perspectiva que se focaliza únicamente en la necesidad de mejorar la "rigurosidad" de lo reportado deja de lado un criterio que resulta a la postre mucho más importante: el de imprimirle relevancia a los debates científicos (Nguyen y Mcllwaine, 2011, p. 15). En este sentido, la cobertura noticiosa de la ciencia resulta muchas veces abstracta para la mayoría de las audiencias (Griffin, Shickle y Moran, 2008; Moran, Shickle, y Richardson, 2008) y, por consiguiente, no logra la suficiente atención e interés por parte del público. Tanto periodistas como científicos intentan compensar este aspecto con estrategias comunicacionales que hacen la ciencia más accesible o su contenido más entretenido, lo que está bien para la comunicación científica, pero resulta inadecuado para el periodismo científico. Este es el reto que aún tienen por delante los periodistas de esta fuente y, en gran medida, la tarea que los científicos deben aceptar como necesaria.

\section{Metodología}

Para examinar esta tesis, hemos adoptado una metodología cualitativa e interdisciplinaria que utiliza estrategias de investigación como las entrevistas semiestructuradas a 52 periodistas en dos países donde el reportaje de la ciencia tiene una larga tradición: el Reino Unido y la India. Las preguntas a los periodistas se centraron en las formas y estilo que usan para comunicar la noticia científica y cómo ellos perciben el rol de las emociones en sus narrativas.

La selección de estos dos países se debió fundamentalmente a la necesidad de examinar esta cobertura en el marco histórico del proyecto de la Ilustración, por lo que las relaciones coloniales y poscoloniales en la formación del periodismo resultaron ser determinantes. La selección de periodistas en ambas sociedades también permitió contrastar la forma en que estos profesionales usan las emociones en la articulación de las noticias científicas. Igualmente 
importante es el contexto populista que deben enfrentar los periodistas en cada uno de los países (ejemplificado por el voto brexit en el Reino Unido y la elección de Narendra Modi como presidente de la India). Siguiendo instrucciones de los comités éticos de la Universidad de Leeds y de la Universidad de Teesside, hemos asegurado el anonimato de los participantes.

Adicionalmente, hemos analizado una muestra de trabajos - una selección de veinte piezas noticiosas - con las técnicas de lectura cerrada. Como el método anterior, la lectura cerrada es una estrategia cualitativa y en ningún momento pretende reflejar una naturaleza general. Como tal, en cambio, estas estrategias cuantitativas sirven para explorar conceptualmente nuestro argumento. Esto nos ha permitido contrastar cómo los valores noticiosos se reflejan en el trabajo final. Cabe explicar que hemos tomado la definición de valores noticiosos, que se refiere a los elementos que el periodista adscribe a lo que reporta y que permite considerar o no un hecho como noticioso (Fuller, 1996; Harcup y O'Neill, 2001; Galtung y Ruge, 1965).

A pesar de que la muestra sea relativamente pequeña y no sea representativa, nos permite ilustrar algunos de los argumentos centrales en este trabajo. Hemos condensado los resultados, dado que el análisis se hizo en inglés. Toda la data transcrita, tanto de las entrevistas como de los ejemplos usados para la lectura cerrada de medios, estará disponible en el repositorio de la
Universidad de Leeds en el Reino Unido hasta el año 2021.

\section{Resultados de campo}

En general, los periodistas, a pesar de sus aspiraciones normativas de objetividad e imparcialidad, usan en ambos países las emociones como una herramienta para vincularse con sus audiencias. Sin embargo, lo hacen de forma muy distinta. Igualmente, es importante notar que la cobertura noticiosa televisiva en el Reino Unido resulta estéticamente más sobria que su contraparte en la India. A pesar de ello y en términos generales, las emociones raramente están en el centro de las narrativas científicas en ninguno de los dos países.

Esto dista de las apreciaciones normativas, ya que la gran mayoría de los entrevistados aseguran que la "rigurosidad periodística" debe estar en el centro de la cobertura noticiosa, pero admiten que el contar la noticia de forma interesante, accesible y entretenida es vital para capturar la atención del público. En otras palabras, aun cuando en sus aspiraciones normativas reclaman una narrativa objetiva, los propios periodistas reconocen que en la práctica es importante incorporar las emociones como parte de sus narrativas noticiosas (aunque no terminen haciéndolo).

El carácter distintivo del periodismo, centrado en el valor de informar objetivamente y de manera imparcial, es compartido por los periodistas en ambos países, algo que concuerda con encuestas mundiales entre estos 
profesionales (WJS, 2018). Esto se refleja también en el análisis de la muestra que subraya que en el Reino Unido y la India predomina la estructura tradicional de la noticia. Sin embargo, mientras en lo referente al uso de las emociones en la articulación de la noticia los periodistas británicos tratan de evitar hacerlo de forma exagerada, sus contrapartes indias son más proclives a él.

Los periodistas de ambos bandos consideran que para contar efectivamente las historias relacionadas con las noticias que se reportan "es esencial que se refieran a "la vida humana'". Como lo sintetiza uno de los periodistas entrevistados:

Un hombre de la Edad de Piedra intentaba advertir a sus hijos que no se acercaran a un tigre, por lo que contaba la historia de un hombre al que se lo comió un tigre. Solían contar historias el uno al otro. Eso es lo que es la televisión... Como especie, nos contamos historias; el homo narrans. (Periodista británico B13 de Sky News)

Esta visión es compartida plenamente por los periodistas de la India, que también resaltan la importancia de narrar la noticia de forma entretenida para atraer al público:

En el periodismo uno no se conecta con noticias. En vez de eso, uno se conecta con las personas y - a su vez- conecta a esas personas entre sí a través de sus emociones. Por ello, la que uno cuenta como una historia noticiosa busca ser un reflejo de lo que la gente ve a través de sus ojos. El buen periodismo no solo informa, sino que también trata de contar historias y relatos. (Periodista indio 11, Doordarshan)

Las entrevistas mostraron, además, un amplio consenso entre los entrevistados en este sentido. Tanto los profesionales británicos como sus homólogos indios concuerdan en que el uso de las emociones en la narración es central para atraer la atención y el interés de las audiencias noticiosas. En palabras de uno de los entrevistados:

Los hechos importan, pero también las secuencias que dejan en la vida de la gente y cómo estos hechos se presentan. Contar historias se ha vuelto mucho más emocional gracias a la multiplicación de emisoras privadas en India. Tienes que tratar de encontrar un caso que refleje el ángulo humano; debes centrar la historia en alguien, un individuo o un grupo de personas, para humanizarlo. Necesitas alguien dispuesto a hablar de sus emociones y no solo de los hechos. Estas historias necesitan, además, controversia, alguien para decir las cosas opuestas. Incluso cuando una historia no es entretenida, uno debe informarla de una manera entretenida. (Periodista británico B19 de Sky News)

Está claro que, a pesar de los tipos ideales normativos que caracterizan al periodista como un individuo que aproxima, produce y disemina la noticia de forma objetiva e imparcial, la apreciación de los propios periodistas es distinta, al menos con respecto a la presentación de la noticia.

Sin embargo, al analizar las historias que los periodistas producen alrededor de las noticias científicas 
podemos observar que el producto final se asemeja más a la apreciación normativa referente a la objetividad e imparcialidad que al reconocimiento de la necesidad de insertar emotividad en las narraciones. En casi todos los casos, los reportajes científicos se presentan, en su mayoría, sin el elemento emotivo. Solo en reportajes de salud, uno observa referencias al impacto humano en mayor extensión. En otras palabras, al analizar las diferentes muestras noticiosas es posible notar que las historias científicas tienden más bien a omitir el elemento humano y concentrarse en informar los hechos. Por ejemplo, en un reportaje titulado "Examinando los planes de transporte hipersónico de China", producido por Andreas Illmer de la BBC (2018), el periodista abre la nota diciendo:

Investigadores chinos han presentado un nuevo concepto de diseño para un avión hipersónico, que según ellos es un gran paso hacia algún día volar de Pekín a Nueva York en solo unas horas. Esta sin duda sería una opción más rápida y quizás más conveniente que las 14 horas actuales.

En ningún momento se habla de los aspectos humanos, de las diásporas chinas o estadounidenses, y cómo esto ayudaría a conectarlas, ni se identifica a los individuos que hicieron posible este desarrollo tecnológico.

Otra historia del mismo período estudiado se refiere a una noticia bajo el título "China lanzará el cohete Long March 5B al espacio en el 2019" de la agencia de noticias india PTI, en The
Times of India del 5 de marzo del 2018; se abre con esta entrada:

El cohete portador de Long March 5B de China, que es capaz de transportar una carga útil de $25000 \mathrm{~kg}$ en órbita terrestre baja, será lanzado al espacio en el 2019, dijo un alto funcionario espacial. El cohete portador se someterá a pruebas este mes en preparación para la primera misión de lanzamiento, y ayudará a llevar el módulo central y los módulos experimentales a la estación espacial de China, señaló un portavoz de la Oficina de Ingeniería Espacial Tripulada de China (CMSEO).

Nuevamente podemos ver que se reportan los hechos sin ningún tipo de relación con lo emotivo o el elemento humano. Este aspecto no se limita a las historias de tecnología, astronomía o exploración espacial. Otras fuentes noticiosas relacionadas con la ciencia presentan narrativas similares. Por ejemplo, una noticia con el título "Pérdida de árboles está empujando a los escarabajos al borde de la extinción", producida por Helen Briggs de la BBC (2018), señala:

La pérdida de árboles en toda Europa está empujando a los escarabajos al borde de la extinción, según un nuevo informe. La Unión Internacional para la Conservación de la Naturaleza evaluó el estado de 700 escarabajos europeos que viven en madera vieja y hueca. Casi una quinta parte (18\%) se encuentra en riesgo de extinción debido a la disminución de los árboles antiguos, de acuerdo con el informe de la Lista Roja Europea de Escarabajos Saproxílicos. Esto los coloca entre los grupos de insectos más 
amenazados de Europa. Los escarabajos saproxílicos desempeñan un papel en los procesos naturales, como la descomposición y el reciclaje de nutrientes. También proporcionan una importante fuente de alimento para aves y mamíferos, y algunos están involucrados en la polinización.

La nota es rigurosa y detalla los hechos de forma cierta, por lo que cumple con las expectativas de la comunidad científica. Sin embargo, con relación a crear narrativas que logren atraer la atención del público, no contiene elementos emocionales que faciliten una vinculación en este sentido. Esta característica se repite casi en su totalidad en la muestra de la India y del Reino Unido, salvo en ocasiones en el área de la salud, donde es más fácil apreciar el elemento humano/emotivo vinculante.

Tanto las entrevistas como la muestra de contenido apuntan también a otro elemento importante: una narrativa explicativa que refleja una racionalidad particular. Con ello queremos decir que las historias que se cuentan en ciencia se hacen desde una perspectiva positivista. Esta perspectiva está dominada por un tono neutral que separa el narrador de los hechos, que enfatiza en la presentación sistemática de estos últimos y que se abstiene de enmarcarlos en un contexto más personal o social.

Esta parece ser una característica particular de la cobertura de la fuente científica. Investigaciones en otras áreas del periodismo han señalado cómo las narrativas periodísticas tienden a evidenciar una cantidad de racionalidades -o lógicas explicativas- que reflejan mucho más las emociones, pese a los estilos y códigos editoriales que demandan objetividad e imparcialidad (Glück, 2016; Peters, 2011; Tumber y Prentoulis, 2003). Este, sin embargo, no es el caso de las noticias sobre ciencia, donde el marco explicativo tiende a ser mucho más restringido y refleja mejor los términos normativos asociados con la objetividad, particularmente con respecto a la comunicación de los hechos. En una historia publicada por la agencia de noticias Indo-Asian News Service (Davis, 2018) con el título de "La primavera llega antes en las regiones polares que en cualquier otro lugar", se puede leer:

El estudio, publicado en la revista en línea Nature Reports, mostró que, por cada 10 grados al norte del ecuador que se mueve, la primavera llega unos cuatro días antes de lo que ocurría hace una década. Este aumento hacia el norte en la tasa de avance de la primavera es aproximadamente tres veces mayor que lo que los estudios previos han indicado. Por ejemplo, en latitudes medias y meridionales, como Los Ángeles, Nueva Orleans o Dallas, el estudio sugiere que la primavera podría llegar apenas un día antes de lo que era hace una década. Más al norte, en Seattle, Chicago o Washington D. C., podría llegar cuatro días antes. Y si vives en el Ártico, podría llegar hasta 16 días antes.

$Y$ en The Indian Express, por la agencia PTI (2 de abril del 2018), se escribe:

Científicos están desarrollando un equipo de anchura atómica que puede 
recibir y transmitir señales en una frecuencia más alta que la que los seres humanos logran escuchar. El equipo Drumhead - como se le denomina - ha sido desarrollado por científicos de la Universidad de Case Western Reserve en Estados Unidos y es un billón de veces más pequeño en términos de volumen y 100000 veces más delgado que el tímpano humano.

Estas notas, además, muestran cómo el periodismo científico tiende a enfocarse en los intereses particulares del Norte Global, presentando racionalidades que suelen ofrecer explicaciones que son distantes de los valores y perspectivas históricas de ciertas comunidades en el Sur Global.

Una de las razones por las que los periodistas tratan de evadir el uso de narrativas emotivas, como lo hacen en otras áreas, es que perciben importantes implicaciones éticas. Estas, según los periodistas entrevistados, crean tensiones. Para autores como Dahlstrom y Ho (2012), existe un amplio consenso alrededor de la narración como técnica de comunicación que solo debe ser empleada para proveer contextos de políticas científicas y no para reportar la ciencia misma. Estos autores enfatizan en que elementos como el propósito subyacente del uso de la narrativa, el tomar en cuenta si la finalidad de la narrativa se orienta a la comprensión o a la persuasión, el cómo esas narrativas pueden comprometer los niveles apropiados de precisión y rigurosidad que se deben mantener, entre otros, entran en contradicción directa con las percepciones de los roles que se consideran apropiados de la comunicación y de los científicos dentro de la democracia (p. 592).

Lo que observamos en nuestra muestra es precisamente una dicotomía entre lo normativo, los retos retóricos a lo normativo y la práctica. Por un lado, la expectativa normativa que, al operar en los parámetros de la objetividad periodística de los reporteros, logra rigurosidad en el trabajo, y el reto de los propios periodistas a esta expectativa, al reconocer la importancia de lo emocional/humano/subjetivo al contar sus historias. Por el otro, una práctica periodística que tiende más bien a cumplir las expectativas normativas y desconocer los retos y críticas que los mismos periodistas plantean a esas expectativas.

\section{Conclusión}

Una de las conclusiones más importantes de este trabajo es que, a pesar de la naturaleza del periodismo científico, su relación con la posverdad es similar a la de otras fuentes noticiosas. Es decir, el periodismo científico no es inmune a la era de los "hechos alternativos", el populismo y las teorías de la conspiración, y al igual que otras fuentes debe enfrentar el declive en los niveles de credibilidad, acceso y aceptación que sufren otras áreas del periodismo moderno. En gran medida, como hemos visto acá, una posible explicación sugiere que la labor del periodismo científico no solo es la de informar, sino también la de vincular al público con los hechos 
noticiosos a través de las historias que cuenta, lo cual presupone un elemento emocional importante en la creación de este vínculo.

El otro problema que el periodismo científico actual enfrenta es que, como fuente noticiosa, se cubre bajo los cánones establecidos por el paradigma de la rigurosidad y de la objetividad/imparcialidad. Esto es ciertamente necesario para asegurar las bases de una práctica profesional que refleje los hechos. Es decir, no hay contradicción. Normativamente, los periodistas aspiran a ser objetivos y a no incorporar emociones, a pesar de que en casi todas las entrevistas reconocen su importancia. Sin embargo, en la práctica - como lo sugiere la lectura cerrada-, lo normativo prevalece y se dejan de lado las emociones.

Esto último es sumamente relevante, ya que, como lo muestran estudios de audiencia, la capacidad que tiene la cobertura científica de vincularse con el público y ganar su atención está altamente constreñida por numerosos elementos relacionados con el estilo y la forma como estas noticias se presentan, y que le dan relevancia o no ante el público.

Adicionalmente, en la era de la posverdad, el periodismo científico tiene que competir por la credibilidad en un espacio público-mediático cada vez más complejo y al cual López-Cantos (2017) denomina, en su análisis de la comunicación pública de la pseudociencia, el ecosistema cognitivo: un ecosistema que está abierto a contenidos fraudulentos en la era de la posverdad. En este ambiente, las emociones tienen un papel determinante en apuntalar la confianza de quienes leen las noticias.

Por tanto, concluimos que la noticia científica no solo enfrenta los problemas relacionados con la falta de rigurosidad y precisión -que acertadamente ha identificado la comunidad científica como problema-, sino que, además, enfrenta el dilema de ser o no relevante para grandes segmentos de las audiencias noticiosas que no ven un vínculo directo con sus propias vidas e intereses y que, asimismo, han perdido credibilidad en el periodismo en general en los tiempos de la posverdad.

\section{Referencias}

Aaronovitch, D., y Langton, J. (2009). Voodoo Histories: The Role of the Conspiracy Theory in Shaping Modern History. Nueva York, NY: Vintage Publishing.

Allcott, H., y Gentzkow, M. (2017). Social Media and Fake News in the 2016 Election. Journal of Economic Perspectives, 31(2), 211-36. doi:10.1257/jep.31.2.211

Anderson, C. W. (2011). Deliberative, Agonistic, and Algorithmic Audiences: Journalism's Vision of its Public in an Age of Audience Transparency. International Journal of Communication, 5(19), 527-549. Recuperado de http://ijoc. org/index.php/ijoc/article/view/884/537 
Badash, L. (2009). A Nuclear Winter's Tale. Science and Politics in the 1980s. Cambridge, MA: MIT Press.

Bakir, V., y McStay, A. (2017). Fake News and The Economy of Emotions: Problems, Causes, Solutions. Digital Journalism, 6(2), 154-175. https://doi.org/10.1080/21670 811.2017.1345645

Bauer, M. W., y Bucchi, M. (Eds.). (2008). Journalism, Science, and Society: Science Communication between News and Public Relations. Abingdon, Oxfordshire: Routledge.

Begg, N., Ramsay, M., White, J., y Bozoky, Z. (1998). Media Dents Confidence in MMR Vaccine. BMJ, 316(7130), 561. https://doi.org/10.1136/bmj.316.7130.561

Benchimol, A. (2015). Before Blackwood's: Scottish Journalism in the Age of Enlightenment. Abingdon, Oxfordshire: Routledge.

Brechman, J., Lee, C. J., y Cappella, J. N. (2009). Lost in Translation? A Comparison of Cancer-Genetics Reporting in the Press Release and its Subsequent Coverage in the Press. Journal of Science Communication, 30(4), 453-474. https://doi. org/10.1177/1075547009332649

Briggs, H. (25 de marzo del 2018). Tree loss pushing beetles to the brink. BBC News. Recuperado de http://www.bbc.com/news/science-environment-43262254

Christians, C. (2015). North-South Dialogues in Journalism Studies. African Journalism Studies, 36(1), 44-50. https://doi.org/10.1080/23743670.2015.1008129

Cohen, B. (2015 [1963]). Press and Foreign Policy. Princeton, NJ: Princeton University Press.

Conway, B. A., Kenski, K., y Wang, D. (2015). The Rise of Twitter in the Political Campaign: Searching for Intermedia Agenda-Setting Effects in the Presidential Primary. Journal of Computer-Mediated Communication, 20(4), 363-380. https://doi. org/10.1111/jcc4.12124

Conway, E. M., y Oreskes, N. (2010). Merchants of Doubt. How a Handful of Scientists Obscured the Truth on Issues from Tobacco Smoke to Global Warming. Nueva York, NY: Bloomsbury Press.

Dahlstrom, M. F., y Ho, S. S. (2012). Ethical Considerations of Using Narrative to Communicate Science. Science Communication, 34(5), 592-61. https:/doi. org/10.1177/1075547012454597

d'Ancona, M. (2017). Post-truth: The New War on Truth and How to Fight Back. Nueva York, NY: Random House.

Davis, U. (5 de marzo del 2018). Spring is springing earlier in polar regions than across the rest of Earth. Phys.org. Indo-Asian News Service. Recuperado de https://phys.org/news/2018-03-earlier-polar-regions-rest-earth.html 
Debarbieux, B., y Rudaz, G. (2015). The Mountain: A Political History from the Enlightenment to the Present. Chicago, IL: University of Chicago Press.

Deuze, M. (2005). What is Journalism? Professional Identity and Ideology of Journalists Reconsidered. Journalism, 6(4), 442-464.https://doi.org/10.1177/1464884905056815

Dunwoody, S. (2014). Science Journalism. Nueva York, NY: Routledge.

Elías, C. (2008). Fundamentos de periodismo científico y divulgación mediática. Madrid: Alianza Editorial.

Ettema, J. S., y Glasser, T. L. (1998). Custodians of Conscience. New York, NY: Columbia University Press.

Feldman, S., Huddy, L., y Marcus, G. E. (2015). Going to War in Iraq: When Citizens and the Press Matter. Chicago, IL: University of Chicago Press.

Fenster, M. (1999). Conspiracy Theories: Secrecy and Power in American Culture. Mineápolis, MN: University of Minnesota Press.

Fowler, R. (2003). Language in the News: Discourse and Ideology in the Press. Abingdon, Oxfordshire: Routledge.

Fuller, J. (1996). News Values: Ideas for an Information Age. Chicago, IL: University of Chicago Press.

Galtung, J., y Ruge, M. H. (1965). The Structure of Foreign News: The Presentation of the Congo, Cuba and Cyprus Crises in Four Norwegian Newspapers. Journal of Peace Research, 2(1), 64-90. https://doi.org/10.1177/002234336500200104

Gee, J. (1999). An Introduction to Discourse Analysis. Theory and Method. Abingdon, Oxfordshire: Routledge.

Glück, A. (2016). What Makes a Good Journalist? Empathy as a Central Resource in Journalistic Work Practice. Journalism Studies, 17(7), 893-903. https://doi.org/10. 1080/1461670X.2016.1175315

Griffin, M., Shickle, D. A., y Moran, N. (2008). European Citizens Opinions on Water Fluoridation. Community Dentistry and Oral Epidemiology, 36(2), 95-102. https:// doi.org/10.1111/j.1600-0528.2007.00373.x

Harcup, T., y O'Neill, D. (2001). What is News? Galtung and Ruge Revisited. Journalism Studies, 2(2), 261-280. http://dx.doi.org/10.1080/14616700118449

Hermida, A. (2012). Tweets and Truth: Journalism as a Discipline of Collaborative Verification. Journalism Practice, 6(5-6), 659-668. https://doi.org/10.1080/1751278 6.2012.667269

Hibberd, M., y Nguyen, A. (2013). Climate Change Communications \& Young People in the Kingdom: A Reception Study. International Journal of Media \& Cultural Politics, 9(1), 27-46. https://doi.org/10.1386/macp.9.1.27_1 
Hjarvard, S. (2008). The Mediatization of Society. Nordicom Review, 29(2), 102-131. https://doi.org/10.1515/nor-2017-0181

Illmer, A. (2 de marzo del 2018). Examining China's hypersonic transport plans. BBC News. Recuperado de www.bbc.co.uk/news/business-43151175

Inglehart, R., y Norris, P. (2016). Trump, Brexit, and the Rise of Populism: Economic Have-Nots and Cultural Backlash. HKS Working Paper No. RWP16-026. Recuperado de https://papers.ssrn.com/sol3/papers.cfm?abstract_id=2818659

Jukes, S. (2013). A Perfect Storm: Journalism Facing Unprecedented Challenges. En K. Fowler-Watt y S. Allan (Eds.), Journalism: New Challenges (pp. 1-18). Recuperado de https://microsites.bournemouth.ac.uk/cjcr/files/2013/10/2013-Journalism-New_ Challenges-Fowler-Watt_and_Allan-v1-01.pdf\#page $=24$

Kalichman, S. C. (2009). Denying AIDS: Conspiracy Theories, Pseudoscience, and Human Tragedy. Berlín: Springer Science \& Business Media.

Linstead, S. A. (2015). The Dark Side of the Open Road: Critical Management Studies and Critical Public Relations. Academy of Management Proceedings, 2015(1). doi:10.5465/AMBPP.2015.16991abstract

López-Cantos, F. (2017). Comunicación pública de la pseudociencia: homeópatas y orgonitas 2.0. Razón y Palabra, 21(96), 355-372. Recuperado de http://www. redalyc.org/html/1995/199551160020/

Lugo-Ocando, J. (2015). Journalists do Live in a Parallel Universe: A Response to Practitioner Critiques of Journalism Academics. Journal of Applied Journalism $\mathcal{E}$ Media Studies, 4(3), 369-379. https://doi.org/10.1386/ajms.4.3.369_1

Lugo-Ocando, J., y Nguyen, A. (2017). Developing News: Global Journalism and the Coverage of "Third World". Abingdon, Oxfordshire: Routledge.

Lundby, K. (Ed.). (2009). Mediatization: Concept, Changes, Consequences. Oxford: Peter Lang.

Luther, C. A., y Miller, M. (2005). Framing of the 2003 US-Iraq War Demonstrations: An Analysis of News and Partisan Texts. Journalism \& Mass Communication Quarterly. 82(1), 78-96. https://doi.org/10.1177/107769900508200106

Martinisi, A., y Lugo-Ocando, J. (2015). Overcoming the Objectivity of the Senses: Enhancing Journalism Practice through Eastern Philosophies. International Communication Gazette, 77(5), 439-455. https://doi.org/10.1177/1748048515586944

Moon, A. (2017). Two-thirds of American adults get news from social media: survey. Reuters. Recuperado de https://uk.reuters.com/article/us-usa-internetsocialmedia/two-thirds-of-american-adults-get-news-from-social-mediasurvey-idUKKCN1BJ2A8 
Moran, N., Shickle, D., y Richardson, E. (2008). European Citizens' Opinions on Immunisation. Vaccine, 26(3), 411-418.https://doi.org/10.1016/j.vaccine.2007.11.001

Murray, C., Parry, K., Robinson, P., y Goddard, P. (2008). Reporting Dissent in Wartime: British Press, the Anti-War Movement and the 2003 Iraq War. European Journal of Communication, 23(1), 7-27. https://doi.org/10.1177/0267323107085836

Neuman, W. R., Guggenheim, L., Mo Jang, S., y Bae, S. Y. (2014). The Dynamics of Public Attention: Agenda-Setting Theory Meets Big Data. Journal of Communication, 64(2), 193-214. https://doi.org/10.1111/jcom.12088

Newman, N., Fletcher, R., Kalogeropoulos, A., Levy, D., y Nielsen, R. K. (2017). Reuters Institute Digital News Report 2017. Recuperado de https://papers.ssrn.com/sol3/ papers.cfm?abstract_id=3026082

Nguyen, A., y McIlwaine, S. (2011). Who Wants a Voice in Science Issues - and Why? A Survey of European citizens and its Implications for Science Journalism. Journalism Practice, 5(2), 210-226. https://doi.org/10.1080/17512786.2010.527544

Oliver, J. E., y Wood, T. J. (2014). Conspiracy Theories and the Paranoid Style(s) of Mass Opinion. American Journal of Political Science, 58(4), 952-966. https://doi. org/10.1111/ajps.12084

Peters, C. (2011). Emotion Aside or Emotional Side? Crafting an "Experience of Involvement" in the News. Journalism, 12(3), 297-316. https://doi. org/10.1177/1464884910388224

Peters, C., y Broersma, M. J. (Eds.). (2013). Rethinking Journalism: Trust and Participation in a Transformed News Landscape. Abingdon, Oxfordshire: Routledge.

Pew Research Center. (2017). Trust in the News Media. Pew Research Centre, Media $\mathcal{E}$ Journalism. Recuperado de http://www.journalism.org/2017/05/10/americansattitudes-about-the-news-media-deeply-divided-along-partisan-lines/ pj_2017-05-10_media-attitudes_a-05/

PTI. (5 de marzo del 2018). China to launch Long March 5B rocket into space in 2019. The Times of India. Recuperado de https://timesofindia.indiatimes.com/ world/china/china-to-launch-long-march-5b-rocket-into-space-in-2019/ articleshow/63172833.cms

PTI. (2 de abril del 2018). Scientists working on new device that could give humans "cat-like" hearing. The Indian Express. Recuperado de http://indianexpress. com/article/technology/science/scientists-working-on-new-device-that-couldgive-humans-cat-like-hearing-5120573/

Rainie, L. (2017). US Public Trust in Science and Scientists [presentación de diapositivas]. Recuperado de http://www.pewinternet.org/2017/06/27/u-spublic-trust-in-science-and-scientists/ 
Rasmussen, D. C. (2014). Contemporary Political Theory as an Anti-Enlightenment Project. En G. Boucher y H. M. Lloyd (Eds.), Rethinking the Enlightenment: Between History, Philosophy, and Politics (pp. 39-59) Boulder, CO: Lexington Books.

Reed, R. (2001). (Un-)Professional Discourse? Journalists' and Scientists' Stories about Science in the Media. Journalism, 2(3), 279-298. https://doi. org/10.1177/146488490100200310

Roudakova, N. (2017). Losing Pravda: Ethics and the Press in Post-Truth Russia. Cambridge: Cambridge University Press.

Russial, J., Laufer, P., y Wasko, J. (2015). Journalism in Crisis? Javnost-The Public, 22(4), 299-312. https://doi.org/10.1080/13183222.2015.1091618

Sánchez Lobato, J., y Hernando, L. A. (2018). La configuración lingüístico-discursiva en el periodismo científico. Madrid: Iberoamericana.

Shermer, M. (1999). The Measure of a Life: Carl Sagan and the Science of Biography. Skeptic, 17(4), 32-39. Recuperado de http://www.theeway.com/skepticc/ newsworthy06.html

Sillesen, L. B., Ip, C., y Uberti, D. (2015). Journalism and the Power of Emotions. Columbia Journalism Review, 54(1), 15. Recuperado de https://www.cjr.org/ analysis/journalism_and_the_power_of_emotions.php

Soronen, A. (2017). Emotional Labour in Magazine Work: Suppressing and Evoking Emotions as Part of Project-Based Teamwork. Journalism Practice, 12(3), 290-307. https://doi.org/10.1080/17512786.2017.1297685

Speed, E., y Mannion, R. (2017). The Rise of Post-Truth Populism in Pluralist Liberal Democracies: Challenges for Health Policy. International Journal of Health Policy and Management, 6(5), 249. https://dx.doi.org/10.15171\%2Fijhpm.2017.19

Speers, T., y Lewis, J. (2004). Journalists and Jabs: Media Coverage of the MMR Vaccine. Communication $\mathcal{E}$ Medicine, 1(2), 171-181. https://doi.org/10.1515/ come.2004.1.2.171

Sturgis, P., y Allum, N. (2004). Science in Society: Re-Evaluating the Deficit Model of Public Attitudes. Public Understanding of Science, 13(1), 55-74. https://doi. org/10.1177/0963662504042690

Tuchman, G. (1972). Objectivity as Strategic Ritual: An Examination of Newsmen's Notions of Objectivity. American Journal of Sociology, 77(4), 660-679. https://doi. org/10.1086/225193

Tumber, H., y Prentoulis, M. (2003). Journalists under Fire: Subcultures, Objectivity, and Emotional Literacy. En D. K. Thussu y D. Freedman, War and the Media: Reporting Conflict (pp. 215-230). Londres: Sage. 
Waisbord, S. (2013). Reinventing Professionalism: Journalism and News in Global Perspective. Hoboken, NJ: John Wiley \& Sons.

Waisbord, S. (2018). The Elective Affinity between Post-Truth Communication and Populist Politics. Communication Research and Practice, 4(1), 17-34, doi:10.1080/22 041451.2018.1428928.

Ward, S. J. A. (2015). The Invention of Journalism Ethics: The Path to Objectivity and Beyond. Kingston, Ontario: McGill-Queen's Press-MQUP.

WJS. (2018). The Worlds of Journalism Study. Recuperado de http://www.world sofjournalism.org

Zelizer, B. (1993). Journalists as Interpretive Communities. Critical Studies in Media Communication, 10(3), 219-237. https://doi.org/10.1080/15295039309366865

Zelizer, B. (2015). Terms of Choice: Uncertainty, Journalism, and Crisis. Journal of Communication, 65(5), 888-908. https://doi.org/10.1111/jcom.12157

Zuckerman, E. (2017). Mistrust, Efficacy, and the New Civics: Understanding the Deep Roots of the Crisis of Faith in Journalism. Knight Commission Workshop on Trust, Media, and American Democracy. Recuperado de https://assets.aspeninstitute. org/content/uploads/2017/07/zuckerman.whitepaper.FINAL_.pdf 